\title{
¿En qué medida utilizan los estudiantes de Física de Bachillerato sus propios errores para aprender? Una experiencia de autorre- gulación en el aula de secundaria
}

\section{To what extent do high school physics students use their own mistakes to learn? An experience on self-regulation in a secondary school classroom}

Ángela Zamora

Universidad Nacional de Educación a Distancia

angzamora@gijon.uned.es

Diego Ardura

Colegio Santo Domingo de Guzmán - FESD (Oviedo)

d.ardura.ovi@fesd.es

RESUMEN • En este artículo presentamos los resultados de una experiencia en autorregulación del aprendizaje en el aula de física de secundaria. El objetivo principal de esta investigación es comprender cómo los estudiantes gestionan la información que les proporciona una prueba escrita sobre sus conocimientos, así como valorar la eficacia de la autorregulación que los estudiantes llevan a cabo a posteriori. Hemos encontrado deficiencias importantes en estos procesos y una relación significativa entre el éxito escolar y estos. Como consecuencia, observamos la necesidad de implementar en el aula estrategias que permitan a los estudiantes no solo el análisis de sus errores, sino también la toma de decisiones adecuadas para utilizarlos como un medio para el aprendizaje.

PALABRAS CLAVE: autorregulación; errores; evaluación formativa; educación secundaria; Física.

ABSTRACT - In this article, we present the results of an experience in self-regulated learning in a secondary school physics classroom. The main goal of this research is to understand how the students manage the information given by a test about their knowledge and also to assess the efficacy of the self-regulatory processes, which students undertake subsequently. We have found serious deficiencies in these processes and a significant relationship between success at school and them. As a result, we point out the need of implementing strategies in the classroom not only to help students analyze their own mistakes but also to use them as a means to learn in the subsequent decision-making process.

KEYWORDS: self-regulation; mistakes; formative evaluation; secondary education; Physics.

Fecha de recepción: diciembre 2012 • Aceptado: marzo 2013 


\section{INTRODUCCIÓN}

El análisis de los principales retos a los que se enfrenta el sistema educativo español ha llevado, siguiendo las directrices europeas y las recomendaciones de la OCDE, a la incorporación de las llamadas competencias básicas como elementos esenciales en la aplicación de la Ley Orgánica de Educación (LOE) (Escamilla, 2008). El éxito de la educación basada en competencias que propone esta ley está supeditado a una modificación significativa en los procesos de enseñanza-aprendizaje (Latorre, 2003; Monereo, 2009). En este nuevo escenario, el profesor -tradicionalmente considerado un mero "transmisor de conocimientos"- debe pasar a actuar como un "mediador" que ayude al alumnado a alcanzar la independencia y autonomía suficientes para que se convierta en el protagonista de su aprendizaje y lo prolongue durante el transcurso de su vida con independencia de su situación personal, académica y social (Cabrerizo, Rubio y Castillo, 2008). Un medio imprescindible para alcanzar este objetivo será el desarrollo en los estudiantes de la competencia para aprender a aprender. Esta competencia, una de las ocho propuestas en la LOE, implica:

... la conciencia, gestión y autocontrol de las propias capacidades y conocimientos desde un sentimiento de eficacia personal, e incluye tanto el pensamiento estratégico como la capacidad de cooperar, de autoevaluarse, y el manejo eficiente de un conjunto de recursos y técnicas de trabajo intelectual, todo lo cual se desarrolla a través de experiencias de aprendizaje conscientes y gratificantes, tanto individuales como colectivas (Cabrerizo, Rubio y Castillo, 2008: 106).

La regulación del aprendizaje es un elemento básico en la adquisición de la competencia para aprender a aprender, ya que una persona competente en este aspecto es capaz de controlar y regular el modo en que aprende para adecuarlo a los objetivos de la tarea que se propone llevar a cabo. En el ańo 1967, Scriven (1967) introduce por primera vez el concepto de evaluación desde el punto de vista de su función reguladora, en lo que se denominó evaluación formativa. El término evaluación formativa se refiere al conjunto de procedimientos empleados por el docente para adaptar sistemáticamente el proceso didáctico a los progresos y dificultades que encuentran los alumnos. Por tanto, este tipo de evaluación tendría como objetivo la regulación del aprendizaje de modo que la formación responda a las peculiaridades de los que aprenden (Jorbá y Sanmartí, 1994). Según Allal (1991), se distinguen tres formas de regulación formativa: i) la regulación interactiva, que se sitúa integrada en la propia acción formativa; ii) la regulación proactiva, que prevé actividades de formación futura, y iii) la regulación retroactiva, donde se programan actividades de refuerzo después de una evaluación puntual al final de una secuencia de enseñanza-aprendizaje.

Aunque tradicionalmente la regulación del aprendizaje ha corrido a cargo del profesor, la necesidad de que los estudiantes dejen de ser simples receptores de la información y asuman un papel más activo y consciente en su aprendizaje ha llevado al desarrollo de una línea de investigación en el campo de la educación conocida como autorregulación del aprendizaje (Suárez y Fernández, 2004). En las dos últimas décadas se ha prestado una atención creciente a la naturaleza, los orígenes y el desarrollo de los procesos de autorregulación de los estudiantes (Zimmerman, 2002). Podríamos definir el aprendizaje autorregulado como "un proceso activo en el cual los estudiantes establecen los objetivos que guían su aprendizaje intentando monitorizar, regular y controlar su cognición, motivación y comportamiento con la intención de alcanzarlos" (Rosário, 2004; Zimmerman, 2000). Por tanto, la autorregulación del aprendizaje es un proceso abierto que implica una actividad cíclica por parte del estudiante. Tiene lugar en tres fases: previa, de realización y de autorreflexión, y cada una de ellas tiene sus correspondientes procesos y subprocesos (Zimmerman, 2000). Esta estructura requiere ajustes continuos debido a las fluctuaciones en los componentes implicados en el proceso: personal, conductual y social. El hecho de que los estudiantes con un alto nivel de autorregulación sean capaces de percibir su aprendizaje como 
un proceso controlable y sistemático tiene implicaciones profundas en su motivación, lo que conlleva que este aspecto esté influenciando al propio proceso (Pintrich y De Groot, 1990; Shunk, 1984; Zimmerman y Paulsen, 1995). Así, cuando las atribuciones causales que efectúan los estudiantes durante su proceso de aprendizaje se dirigen a los procesos estratégicos que se perciben como mejorables, el alumno incrementa su sensación de auto-eficacia, con el correspondiente incremento del interés intrínseco por la tarea escolar (Núñez, Solano, González-Pienda y Rosário, 2006). Esto ha llevado a la aparición de un interés creciente por la autorregulación del aprendizaje incluso a nivel universitario. Por ejemplo, recientemente se ha sugerido (Hernández-Pina, Sales y Cuesta, 2010) la conveniencia de implantar programas de autorregulación del aprendizaje en los estudios universitarios de grado. El $80 \%$ de los estudiantes valoran positivamente la incorporación explícita de este tipo de programas y el $20 \%$ de ellos consideran conveniente la sensibilización del profesorado en este tema para que se produzca una mejora de los procesos evaluativos.

La gestión de los errores durante el proceso de enseñanza-aprendizaje es, obviamente, fundamental para la regulación del aprendizaje. Astolfi (1999) propone que los profesores deben dejar de considerar los errores como elementos sancionables para aprovechar la oportunidad de usarlos como instrumentos educativos de modo estratégico. Que tanto el alumnado como el profesorado implicado sean capaces de comprender el origen de los errores conduciría paulatinamente a la mejora del proceso de regulación que tiene lugar en las aulas. Por tanto, parece básico un cambio en el estatus del error en la escuela (Nunziati, 1990). En ese contexto, el éxito de los estudiantes estaría relacionado no con la ausencia de errores, sino con su capacidad para detectarlos y corregirlos, lo que facilitaría la laboriosa reconstrucción que implica la apropiación de los conocimientos (Sanmartí, 2007). El análisis de los errores que comenten los estudiantes durante su aprendizaje ha recibido una atención considerable en las últimas décadas. Tanto en el contexto de las matemáticas como en el de las ciencias naturales se han llevado a cabo estudios sistemáticos con el fin de explicar y clasificar las equivocaciones de los estudiantes, así como comprender su origen y los factores que los desencadenan (Buteler, Coleoni, y Gangoso, 2008; Mammino, 2002). Sin embargo, no son muchos los estudios en los que se analiza cómo aprenden los estudiantes a partir de sus propios errores. Recientemente, Mason y Singh (2010) han investigado cómo los estudiantes de último ańo del grado en Física en una universidad estadounidense utilizan sus errores como fuente de aprendizaje. Sorprendentemente, un número considerable de ellos no son capaces de usar de modo automático los errores cometidos como una oportunidad para aprender. Está comprobado que los estudiantes que tienen éxito en la escuela disponen de la capacidad para identificar los errores y corregirlos. Yerushalmi, Cohen, Mason y Singh (2012) se interesaron por conocer la capacidad de autodiagnóstico de una muestra de estudiantes universitarios. Sus resultados están de acuerdo con los encontrados previamente por Mason y Singh (2010), ya que se pone de manifiesto que los únicos estudiantes que detectaron y describieron en cierto grado sus errores fueron aquellos que recibieron un mayor grado de orientación en la tarea de autodiagnóstico. Sin embargo, y de acuerdo con lo expuesto anteriormente, muchos estudiantes no desarrollan esa capacidad de manera espontánea, por lo que se hace necesario implementar estrategias en el aula que les permitan mejorar en estos aspectos que contribuirán considerablemente a desarrollar su capacidad de autorregulación (Núñez y col., 2006; Sanmartí, 2007).

En este artículo se presentan los resultados de una experiencia llevada a cabo en un aula de física de segundo curso de bachillerato y que persiguió los siguientes objetivos:

- Observar el proceso de detección y análisis de sus propios errores por parte de los estudiantes.

- Analizar el proceso de autorregulación retroactiva a través de la propuesta de trabajo que realizan los estudiantes sin la intervención del profesor.

- Evaluar la eficacia didáctica de la experiencia, a partir de la evaluación llevada a cabo por el profesor, así como a través de una autoevaluación de los propios estudiantes. 


\section{METODOLOGÍA}

La investigación se llevó a cabo en una clase de segundo de bachillerato en un colegio español concertado. El número total de alumnos participantes en la experiencia fue 14 ( 7 chicas y 7 chicos). Se seleccionó un grupo muy heterogéneo en cuanto a su rendimiento escolar en la asignatura de Física con el fin de poder establecer consecuencias relevantes tanto en el caso de estudiantes con alto rendimiento como para aquellos en los que se manifiestan distintos grados de fracaso escolar. El profesor encargado de llevar a cabo la experiencia en el aula fue uno de los investigadores que firman este artículo.

La experiencia se desarrolló durante la aplicación de la unidad didáctica de gravitación y tuvo una duración aproximada de un mes. Para desarrollar la unidad se utilizó una metodología expositiva acompañada del trabajo individual de los alumnos sobre las tareas asignadas por el profesor y de la posterior corrección de los ejercicios en el aula para todo el grupo. En la tabla 1 se recogen los quince objetivos didácticos que se trabajaron durante la unidad. Para elaborarlos se tomó como referencia el currículo oficial para la asignatura de Física II regulado en el RD 1467/2007, de 2 de noviembre.

Una vez finalizadas las explicaciones de la unidad, se planteó a los alumnos una prueba escrita consistente en dos preguntas teóricas y tres prácticas que tenían como objetivo evaluar el grado de asimilación de los contenidos de esta. En concreto, se plantearon cinco preguntas basadas respectivamente en los objetivos 01, 02, 07, 11 y 14 de la unidad (tabla 1). Esta prueba sirvió para dos propósitos: en primer lugar, el profesor valoró, basándose en las respuestas de los alumnos, el grado de consecución de los cinco objetivos alcanzados por cada alumno. En segundo lugar, la prueba se utilizó en la siguiente sesión de clase para provocar la búsqueda de errores y la propuesta, a partir de ellos, de la tarea de autorregulación por parte de los alumnos. De acuerdo con sugerencias de trabajos anteriores (Butler, 1987), la prueba se devolvió sin la calificación otorgada por el profesor y, con el fin de no perturbar la observación de la localización y descripción de los errores por parte de los alumnos, no incluyó ninguna nota escrita ni corrección que pudiera dirigir a los estudiantes en esta actividad. Así mismo, a lo largo de la experiencia, el profesor siguió escrupulosamente el procedimiento que se describe en este apartado, evitando así cualquier interferencia en la tarea de los estudiantes que pudiera perturbar los resultados de este estudio.

Tabla 1.

Objetivos específicos de la unidad didáctica

01. Conocer el desarrollo histórico de las teorías sobre el sistema solar hasta Kepler.

02. Enunciar y demostrar las leyes de Kepler reconociendo su origen empírico.

03. Aplicar las leyes de Kepler a la resolución de problemas.

04. Definir qué se entiende por momento angular.

05. Calcular el momento angular en casos concretos.

06. Enunciar el teorema de conservación del momento angular.

07. Aplicar el teorema de conservación del momento angular a la resolución de problemas de órbitas elípticas.

08. Enunciar la Ley de Gravitación Universal de Newton (LGUN).

09. Conocer el problema de la determinación experimental de $\mathrm{G}$.

10. Comprender la síntesis de Newton que unificó la explicación para los movimientos observados cerca de la superficie del planeta y los movimientos de los cuerpos celestes.

11. Aplicar la LGUN a la resolución de ejercicios que involucren planetas o satélites.

12. Determinar el periodo de rotación de la Luna tal y como lo hizo Newton por primera vez (a partir del radio de la Tierra, la distancia Tierra-Luna y la aceleración de la gravedad en la Tierra).

13. Determinar la velocidad tangencial que debe tener un objeto lanzado en las proximidades de la Tierra para que describa una circunferencia completa sin tocar el suelo.

14. Aplicar el principio de superposición al cálculo de la fuerza neta ejercida sobre una masa por un conjunto de masas.

15. Resolver problemas en los que intervengan satélites geoestacionarios, empleando la segunda ley de Newton. 
En este momento se les solicitó a los alumnos que completasen las siguientes tareas en unos registros que previamente se les entregaron:

Paso I. Se les pidió a los estudiantes que localizaran y describieran a fondo los errores que habían cometido en cada pregunta. Para esta tarea, que cada estudiante llevó a cabo individualmente, se animó a los alumnos a utilizar sus notas de clase, el libro de texto o cualquier otro material. Este trabajo se realizó individualmente. En este punto y para facilitar la reflexión sobre las respuestas a los ejercicios prácticos, se proporcionaron únicamente los resultados numéricos finales de los ejercicios.

Paso II. Una vez que los alumnos registraron por escrito lo que pensaban que eran sus errores, se les suministró una lista de criterios de corrección en la que se indicaban detalladamente los distintos aspectos que debía incluir cada respuesta correcta (anexo 1). De nuevo, se solicitó a los alumnos la misma tarea que en el paso anterior: detectar y describir sus errores, empleando en este caso la nueva información suministrada por el profesor.

Paso III. Finalmente, se solicitó a los alumnos que hicieran una propuesta de autorregulación retroactiva. Esta propuesta consistió en una lista de tareas que deberían trabajar, en el espacio de una semana, sobre aquellos aspectos en los que considerasen que debían mejorar para una óptima adquisición de los objetivos de aprendizaje en cuestión. Además, debían indicar el tiempo que le dedicarían al trabajo de cada objetivo. Para ello, se le suministró a cada alumno una serie de 50 enunciados de diversas cuestiones y problemas, no trabajados previamente, sobre los contenidos de la unidad. El profesor se ofreció a corregir las producciones de los alumnos resultantes de su propuesta de autorregulación, pero no intervino en ningún momento en la confección de esta.

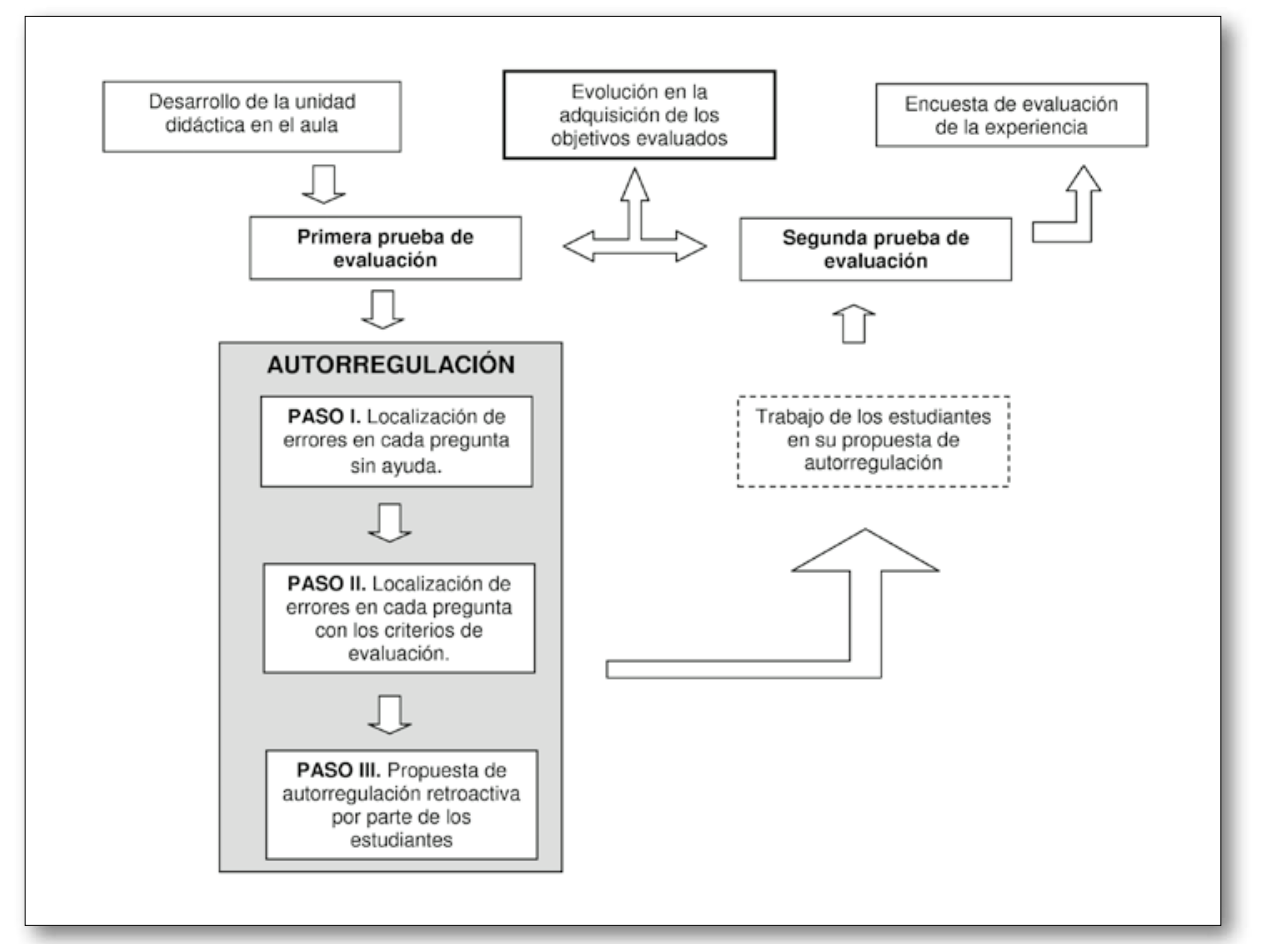

Fig. 1. Diagrama de flujo del proceso seguido en la experiencia

Tras un periodo de dos semanas se llevó a cabo otra prueba, en la que, sin conocimiento previo de los alumnos, se presentaron cinco preguntas sobre los mismos objetivos de aprendizaje que se evaluaron en la primera prueba, con el fin de observar la influencia del trabajo autorregulado. Por último, se pasó a los alumnos una encuesta para evaluar la acción formativa. 


\section{RESULTADOS Y DISCUSIÓN}

En este apartado se presentan en primer lugar los resultados del proceso de detección de errores por parte de los estudiantes. A continuación, el análisis de su propuesta de autorregulación retroactiva y la comparación del rendimiento en cada una de las dos pruebas. Finalmente, se incluyen los resultados de la encuesta de evaluación de la actividad que se pasó a los estudiantes.

\section{A) El proceso de detección de errores por parte de los estudiantes}

\section{A.1. Cuestiones teóricas}

En lo referente a las cuestiones teóricas, cuando se solicitó a los estudiantes la descripción de sus errores en la primera prueba, en primera instancia sin ninguna ayuda, su análisis fue muy impreciso. Prácticamente no hubo ningún alumno que describiese sus errores de una manera detallada y completa. Son habituales descripciones como "he tenido poca claridad en la respuesta" (alumno 8) o "me ha faltado mucha información y algo de lo que he puesto está confundido" (alumna 9). En ocasiones la reflexión es simplemente un parafraseo de la pregunta, como en el caso del alumno 1, que indicó como error en la primera pregunta: "no supe escribir la influencia [del movimiento retrógrado] en el desarrollo de la astrofísica". Otros alumnos, con fallos claros en sus respuestas, dejaron el registro en blanco. Curiosamente, y aunque se les sugirió en varias ocasiones a los estudiantes la posibilidad de consultar sus apuntes y/o el libro de texto, se observó que, en general, no recurrieron a esas herramientas.

La utilización, en el segundo paso de la actividad, de la lista de criterios de corrección mejoró significativamente la localización e interpretación de los errores, así como la descripción de estos, especialmente en las preguntas teóricas. Por ejemplo, el alumno 11, cuando evaluó su ejercicio sin ayuda, describió su error en la primera pregunta como: "no expliqué la evolución en el tiempo de la explicación del movimiento retrógrado”. Sin embargo, al trabajar con ayuda de los criterios transformó esa descripción en: "no expliqué cómo justifican los modelos de Ptolomeo y Copérnico el movimiento retrógrado. Además, no introduje los conceptos epiciclo y deferente”.

\section{A.2. Ejercicios prácticos}

En cuanto al análisis de los ejercicios prácticos, el resultado de la experiencia es similar: la utilización de los criterios de corrección ha facilitado en gran medida, a los estudiantes, la detección y atribución causal de los errores. Sin esta información, los estudiantes se fijaron casi exclusivamente en el resultado final y las correspondientes descripciones de los errores consistieron básicamente en decir si el resultado final del problema era correcto o no, y las equivocaciones se atribuyeron principalmente a "errores de cálculo" cuando en muchos casos existían problemas conceptuales. La utilización de los criterios de corrección permitió a los alumnos una descripción más precisa. Por ejemplo, el alumno 2 pasó de la descripción: "en la pregunta 5 no he sabido usar bien los vectores" a profundizar en la cuestión, haciendo un análisis más detallado, como es: "no supe hacer la descomposición de las fuerzas ni aplicar el principio de superposición".

Durante esta fase de la experiencia, hemos podido comprobar la relevancia de la competencia en comunicación lingüística a la hora de describir los errores, algo que podría influir en la representación mental que los estudiantes se hacen de estos. Es importante destacar por último que existe una clara relación entre la bondad en la descripción de los errores y los resultados alcanzados por los estudiantes en la prueba: cuanto más experto es el estudiante en la materia, mayor facilidad tiene para encontrar y describir sus errores tanto cuando cuenta con el guion de criterios de corrección, como cuando no utiliza esa información. 


\section{B) Análisis de la propuesta de autorregulación retroactiva}

De acuerdo con Jorbá y Sanmartí (1994), las formas de regulación efectivas deben ser actividades que se adapten al tipo de dificultad y al estudiante que la tiene. Por esta razón, la elección consciente por parte de cada estudiante de las actividades que debe realizar sería fundamental para conseguir la autorregulación retroactiva de su aprendizaje. Para observar este proceso, se pidió a los estudiantes que, en función de los errores detectados, propusiesen unas actividades para favorecer su autorregulación retroactiva, de modo que les permitiesen trabajar específicamente en aquellos aspectos que cada uno necesitaba a tenor de sus resultados en la primera prueba. Además de solicitar unas acciones específicas para superar cada error, se dio la posibilidad a los estudiantes de que incluyesen actividades que les pareciesen interesantes para trabajar aspectos de la unidad que no se evaluaban en la prueba o que no estaban relacionados directamente con sus errores. De aquí en adelante llamaremos trabajo extra a estas últimas tareas.

En la tabla 2 se presentan los resultados de la evaluación de la adecuación de la propuesta de cada alumno. En la primera columna se indica el rendimiento $(\mathrm{R})$ en la primera prueba en una escala de A a D (A: Muy bueno; B: Bueno; C: Normal; D: Bajo). En las cinco columnas siguientes se indica la valoración del profesor sobre la adecuación del trabajo de autorregulación retroactiva. El docente situó el trabajo planeado para mejorar en cada error en uno de los siguientes niveles de acuerdo con su magnitud y los conocimientos actuales del estudiante evaluado en particular:

- Nivel 1. El trabajo propuesto resulta insuficiente para la regulación de los errores.

- Nivel 2. El trabajo propuesto resulta adecuado para la regulación de los errores.

- Nivel 3. El trabajo propuesto resulta excesivo para la regulación de los errores.

Finalmente, la última columna se refiere al trabajo extra propuesto por el alumno medido en la escala referida anteriormente. Se señalan en negrita los alumnos que le presentaron al profesor la tarea planificada una semana después de elaborar la propuesta. El resto de los alumnos planificaron la propuesta, pero no la presentaron.

Tabla 2 .

Evaluación de la adecuación del trabajo de autorregulación propuesto por los estudiantes

\begin{tabular}{|c|c|c|c|c|c|c|c|}
\hline & & \multicolumn{7}{|c|}{ PREGUNTAS } & \\
\hline Alumnos/as & R & I & II & III & IV & V & EXTRA \\
\hline 01 & C & 1 & 2 & 1 & 2 & 1 & - \\
\hline 02 & C & 2 & 1 & 1 & 1 & 2 & - \\
\hline 03 & A & 2 & 2 & 2 & 2 & 2 & 3 \\
\hline 04 & B & 2 & 2 & 2 & 2 & 1 & 2 \\
\hline 05 & B & 1 & 2 & 2 & 2 & 2 & 2 \\
\hline 06 & C & 1 & 1 & 2 & 2 & 1 & - \\
\hline 07 & D & 1 & 1 & 1 & 1 & 1 & - \\
\hline 08 & B & 2 & 1 & 2 & 2 & 1 & - \\
\hline 09 & A & 2 & 2 & 2 & 1 & 1 & - \\
\hline 10 & A & 2 & 2 & 2 & 2 & 3 & 3 \\
\hline 11 & D & 1 & 2 & 2 & 1 & 1 & 3 \\
\hline 12 & D & 2 & 2 & 1 & 1 & 1 & 3 \\
\hline 13 & B & 1 & 2 & 3 & 3 & 2 & - \\
\hline 14 & D & 1 & 1 & 1 & 1 & 1 & - \\
\hline
\end{tabular}


Las mayores dificultades que encontraron los alumnos en el proceso de autorregulación tuvieron lugar en lo referente a la propuesta de mejora de los contenidos teóricos. El hecho de no haber profundizado lo suficiente en la descripción de los errores en este tipo de preguntas puede ser una de las causas. Han sido muy frecuentes propuestas muy vagas y excesivamente generales como para actuar específicamente sobre los errores detectados, como por ejemplo: "debo estudiar bien la teoría, comprendiéndola mejor" (alumno 2) o "hacer más demostraciones que pueda encontrar en los apuntes" (alumno 10). Es destacable la ausencia de mención a recursos específicos o técnicas de trabajo que pudieran facilitar el aprendizaje. Tan solo la alumna 9 mencionó sobre la pregunta 1 que "al ser tantos modelos, para reconocer bien las diferencias entre unos y otros debería hacer un esquema por autor para distinguir bien la teoría en relación a quién la propuso".

Tabla 3.

Comparativa de la evolución de los resultados valorados en una escala de Likert del 1 al 4 de la primera prueba (1) a la segunda (2)

\begin{tabular}{|c|c|c|c|c|c|c|c|c|c|c|c|c|}
\hline & \multicolumn{2}{|c|}{ TOTAL } & \multicolumn{2}{l|}{ PREGUNTA 1 } & \multicolumn{2}{l|}{ PREGUNTA 2 } & \multicolumn{2}{l|}{ PREGUNTA 3 } & \multicolumn{2}{l|}{ PREGUNTA 4 } & \multicolumn{2}{l|}{ PREGUNTA 5 } \\
\hline Alumno & 1 & 2 & 1 & 2 & 1 & 2 & 1 & 2 & 1 & 2 & 1 & 2 \\
\hline 01 & 10 & 12 & 2 & 2 & 2 & 3 & 2 & 2 & 3 & 4 & 1 & 1 \\
\hline 02 & 11 & 8 & 1 & 1 & 2 & 2 & 3 & 1 & 3 & 3 & 2 & 1 \\
\hline 03 & 16 & 19 & 4 & 4 & 3 & 4 & 2 & 4 & 4 & 4 & 3 & 3 \\
\hline 04 & 15 & 15 & 2 & 3 & 2 & 3 & 4 & 4 & 4 & 2 & 3 & 3 \\
\hline 05 & 17 & 18 & 4 & 4 & 4 & 4 & 3 & 4 & 2 & 3 & 4 & 3 \\
\hline 06 & 14 & 17 & 2 & 4 & 3 & 3 & 3 & 4 & 2 & 3 & 4 & 3 \\
\hline 07 & 7 & 6 & 1 & 1 & 2 & 2 & 1 & 1 & 2 & 1 & 1 & 1 \\
\hline 08 & 13 & 18 & 2 & 4 & 3 & 4 & 3 & 4 & 2 & 4 & 3 & 2 \\
\hline 09 & 15 & 19 & 3 & 4 & 3 & 4 & 3 & 4 & 3 & 3 & 3 & 4 \\
\hline 10 & 16 & 20 & 3 & 4 & 3 & 4 & 3 & 4 & 4 & 4 & 3 & 4 \\
\hline 11 & 8 & 12 & 2 & 1 & 2 & 2 & 1 & 4 & 2 & 4 & 1 & 1 \\
\hline 12 & 6 & 5 & 1 & 1 & 2 & 1 & 1 & 1 & 1 & 1 & 1 & 1 \\
\hline 13 & 14 & 14 & 1 & 1 & 3 & 3 & 3 & 4 & 4 & 3 & 3 & 3 \\
\hline 14 & 6 & 6 & 1 & 1 & 2 & 2 & 1 & 1 & 1 & 1 & 1 & 1 \\
\hline
\end{tabular}

$\mathrm{Al}$ igual que en el caso de la detección de los errores, aquellos alumnos que mejor resultado obtuvieron en la prueba fueron los que hicieron una propuesta de autorregulación más adecuada de acuerdo con su nivel. Sin embargo, hemos detectado en estos mismos alumnos una tendencia a proponer un volumen de trabajo excesivo (para el tiempo de una semana del que iban a disponer), especialmente en el caso de la propuesta que hicieron de trabajo no relacionado con las preguntas que se les plantearon en la prueba (trabajo extra). Este hecho también nos indicaría un defecto en su capacidad para autorregularse, en este caso por exceso. Algunos de estos alumnos propusieron actividades que resultaban claramente redundantes para su nivel de conocimientos y, por tanto, poco aprovechables para reforzar su aprendizaje en la unidad. También se dio el caso de una propuesta excesiva para el plazo de una semana en algunos alumnos de los que encontraron grandes dificultades en la primera prueba. Si bien en estos casos los alumnos necesitaban un trabajo intensivo, su propuesta era muy difícil de llevar a cabo en una semana. De hecho, solo la mitad de los alumnos entregaron a tiempo lo que habían proyectado y, en todos los casos, se trataba de alumnos que habían tenido un buen rendimiento en la prueba, con la excepción del caso del alumno 2, como se puede ver en la tabla 2. 


\section{C) Evaluación de la actividad por parte del profesor: comparación entre las dos pruebas}

Para evaluar la influencia de la actividad en el aprendizaje de los contenidos de la unidad, se propuso una segunda prueba dos semanas después de que tuviera lugar la primera y cuando el ciclo de autorregulación se había completado. En esta prueba, y sin conocimiento previo de los alumnos, se propusieron cinco preguntas cuya finalidad fue evaluar la evolución en la consecución de los objetivos didácticos trabajados durante la experiencia.

En la tabla 3 se incluyen las calificaciones obtenidas por cada alumno en cada una de las dos pruebas y el correspondiente desglose de las puntuaciones que obtuvieron en cada pregunta. Como se puede apreciar en esta tabla, ocho de los catorce alumnos (alumnos 1, 3, 5, 6, 8, 9, 10 y 11) mejoraron su resultado inicial en la segunda prueba. Cinco de estos alumnos habían entregado las actividades de autorregulación y tres de ellos no. Tres de los estudiantes bajaron su rendimiento (alumnos 2, 7 y 12) con respecto a la primera prueba. Tan solo uno de estos tres, el alumno 2, había presentado el trabajo de autorregulación. Por último, otros tres (alumnos 4, 13 y 14) mantuvieron su rendimiento en las dos pruebas y, de nuevo, solo la alumna 4 había completado su propuesta de actividades. En la figura 2 se pueden ver las diferencias de puntuación total entre las dos pruebas para cada uno de los estudiantes.

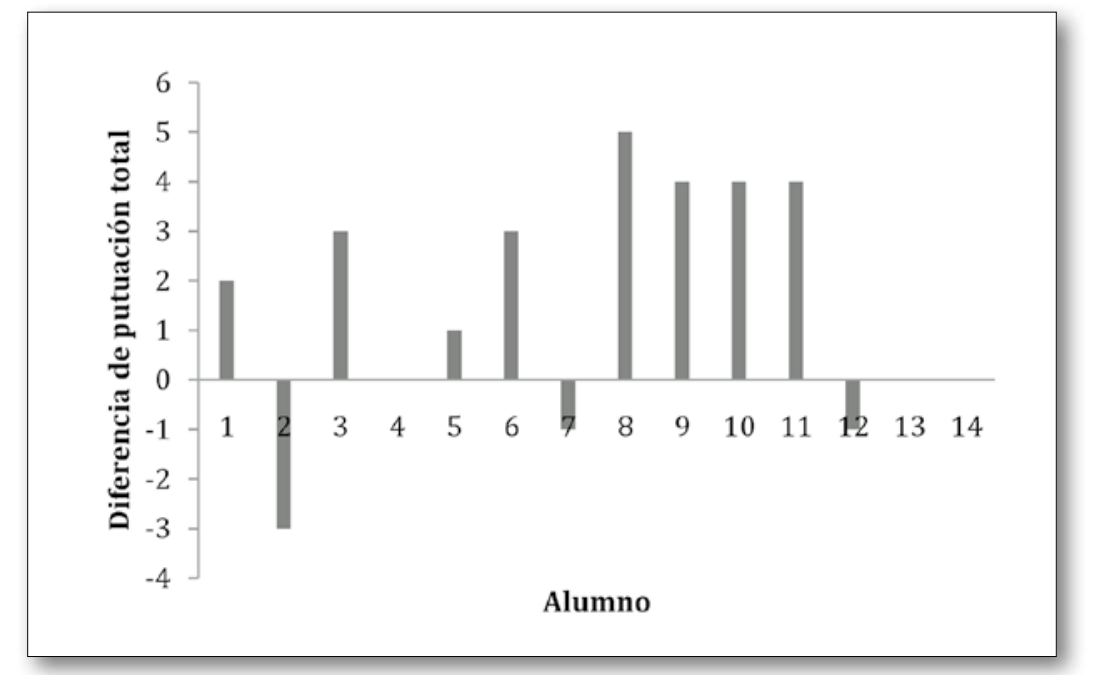

Fig. 2. Diferencias de puntuación total entre la segunda y la primera prueba para todos los alumnos

Para valorar la eficacia de la autorregulación en las distintas preguntas, se compararon las dos pruebas analizando la frecuencia con la que los alumnos mejoraron o empeoraron su rendimiento en cada una de ellas (tabla 4). En líneas generales ha existido una mejoría en el 37\% de las preguntas y un empeoramiento en el $14 \%$ de ellas. En la pregunta 3 un total de ocho respuestas han sido mejores que en la primera prueba. A continuación se sitúan las preguntas 2 y 1 , con seis y cinco respuestas mejoradas en la segunda prueba. En la pregunta 4 fueron cinco los alumnos que mejoraron aunque tres empeoraron sus respuestas. Finalmente, en el ejercicio 5 se puso de manifiesto un empeoramiento, ya que, aunque dos personas incluyeron una respuesta más satisfactoria, otras cuatro la empeoraron. Por tanto, en líneas generales se ha producido una mejoría más significativa en las preguntas teóricas o teórico-prácticas que en los problemas numéricos. 
Tabla 4.

Resumen de las mejoras y empeoramientos en la comparación de la segunda prueba con la primera para cada uno de los ejercicios propuestos en ellas

\begin{tabular}{|l|c|c|c|}
\hline & MEJORÍA & EMPEORAMIENTO & SIN VARIACIÓN \\
\hline Pregunta 1 & 5 & 1 & 8 \\
\hline Pregunta 2 & 6 & 1 & 7 \\
\hline Pregunta 3 & 8 & 1 & 5 \\
\hline Pregunta 4 & 5 & 3 & 6 \\
\hline Pregunta 5 & 2 & 4 & 8 \\
\hline TOTAL & 26 & 10 & 34 \\
\hline PORCENTAJE & $37 \%$ & $14 \%$ & $49 \%$ \\
\hline
\end{tabular}

\section{D) Evaluación de la experiencia por parte de los estudiantes}

Al finalizar la actividad se pasó una encuesta a los alumnos que ha permitido conocer sus impresiones sobre aspectos como la utilidad de la autorregulación en general (preguntas 1 a 3), la valoración de la experiencia que se presenta en este trabajo (preguntas 4 a 7) o las dificultades que han encontrado en el proceso de localización de errores y en la propuesta de trabajo de autorregulación retroactiva (preguntas 8 a 11). Por último, se consultó sobre el grado de implicación de cada alumno en la experiencia. Se utilizó una escala de Likert del 1 al 4 (tabla 5).

Más de la mitad de los estudiantes considera bastante útil o muy útil aumentar su capacidad para autorregularse de cara a afrontar sus estudios posteriores. La mitad de los encuestados manifiesta que sería necesario recibir ayuda para aumentar sus habilidades de autorregulación y el 79\% lo incorporaría a la metodología que se aplica en el aula. Los alumnos que rechazan esta opción son mayoritariamente aquellos que, según los resultados de esta experiencia, tienen suficiente capacidad para autorregular su aprendizaje.

Tabla 5.

Resultados de la encuesta que se pasó a los alumnos

\begin{tabular}{|c|c|c|c|c|}
\hline & 1 & 2 & 3 & 4 \\
\hline $\begin{array}{l}\text { 1. Pienso que aumentar mi capacidad para autorregularme me resultará útil } \\
\text { en mis estudios posteriores. }\end{array}$ & $7,1 \%$ & $35,8 \%$ & $50,0 \%$ & $7,1 \%$ \\
\hline $\begin{array}{l}\text { 2. Sería necesario que recibiésemos más ayuda en las clases para aumentar } \\
\text { nuestra capacidad de autorregularnos. }\end{array}$ & $28,6 \%$ & $28,6 \%$ & $28,6 \%$ & $14,2 \%$ \\
\hline 3. Me parece útil que el método se incorpore a las clases cuando sea posible. & $7,1 \%$ & $14,3 \%$ & $64,3 \%$ & $14,3 \%$ \\
\hline $\begin{array}{l}\text { 4. La experiencia me ha ayudado entender mejor cuáles eran los objetivos de } \\
\text { la unidad. }\end{array}$ & $0 \%$ & $35,7 \%$ & $50,0 \%$ & $14,3 \%$ \\
\hline $\begin{array}{l}\text { 5. El proceso de autorregulación me ha permitido mejorar mi nivel en los } \\
\text { objetivos trabajados y/o corregir errores. }\end{array}$ & $14,3 \%$ & $42,8 \%$ & $28,6 \%$ & $14,3 \%$ \\
\hline $\begin{array}{l}\text { 6. La experiencia de autorregulación me ha parecido interesante en líneas } \\
\text { generales. }\end{array}$ & $21,4 \%$ & $28,6 \%$ & $28,6 \%$ & $21,4 \%$ \\
\hline $\begin{array}{l}\text { 7. La experiencia me ha permitido fijarme y profundizar más en los errores } \\
\text { que cuando se sigue el procedimiento habitual. }\end{array}$ & $7,2 \%$ & $35,7 \%$ & $35,7 \%$ & $21,4 \%$ \\
\hline $\begin{array}{l}\text { 8. El hecho de tener que autoevaluarme me ayuda a mejorar mi rendimien- } \\
\text { to }\end{array}$ & $0 \%$ & $35,7 \%$ & $50,0 \%$ & $14,3 \%$ \\
\hline 9. Me resulta difícil encontrar mis errores. & $21,4 \%$ & $28,6 \%$ & $28,6 \%$ & $21,4 \%$ \\
\hline
\end{tabular}




\begin{tabular}{|c|c|c|c|c|}
\hline & 1 & 2 & 3 & 4 \\
\hline $\begin{array}{l}\text { 10. Me resulta difícil seleccionar las actividades adecuadas para superar los } \\
\text { errores. }\end{array}$ & $0 \%$ & $7,1 \%$ & $50,0 \%$ & $42,9 \%$ \\
\hline $\begin{array}{l}\text { 11. El hecho de que yo mismo decida qué hacer para corregir los errores me } \\
\text { parece más adecuado que el hecho de que el profesor nos ponga una tarea } \\
\text { única para toda la clase. }\end{array}$ & $0 \%$ & $28,6 \%$ & $57,1 \%$ & $14,3 \%$ \\
\hline 12. ¿En qué medida me he involucrado en la experiencia? & $14,3 \%$ & $35,7 \%$ & $28,6 \%$ & $21,4 \%$ \\
\hline
\end{tabular}

El $64 \%$ de los estudiantes manifiesta que la experiencia que presentamos en este artículo les ha ayudado a reflexionar y entender mejor cuáles eran los objetivos didácticos de la unidad, así como a fijarse y profundizar más en los errores que cuando se emplean los métodos habituales. Un $43 \%$ de los alumnos indican que esta actividad les ha permitido mejorar en su rendimiento en cuanto a los contenidos de esta unidad. La experiencia le ha resultado interesante, en líneas generales, a casi a la mitad de los alumnos. El 64\% de los alumnos considera que han mejorado su rendimiento gracias al proceso de autoevaluación que conlleva la experiencia.

La mitad de los estudiantes indica que ha tenido un grado de implicación elevado en la experiencia. En la tabla 6 podemos observar que existe una cierta relación entre el grado de implicación de los alumnos y su mejoría global en la segunda prueba. Así, de los siete alumnos que se han implicado bastante o mucho en la actividad, cinco han mejorado en los contenidos que han trabajado a partir de sus errores. Por otro lado, los dos únicos alumnos que han manifestado no haber seguido la actividad en absoluto no han conseguido ninguna mejoría.

Tan solo dos estudiantes manifiestan en la encuesta que no tienen ninguna dificultad para encontrar los errores. Del resto, hasta seis alumnos encuentran bastantes o muchas dificultades en esta tarea. Además, la mitad de los estudiantes reconocieron tener bastantes o muchas dificultades a la hora de elaborar la propuesta de autorregulación. Tan solo tres de ellos indican que llevaron a cabo esta tarea sin encontrar obstáculos.

Tabla 6.

Comparación entre el grado de implicación

de los estudiantes y la mejoría que demostraron en la segunda prueba

\begin{tabular}{|c|c|c|}
\hline Grado de implicación & Número de alumnos & $\begin{array}{c}\text { Número de alumnos } \\
\text { que mejoran en la segunda prueba }\end{array}$ \\
\hline 1 & 2 & 0 \\
\hline 2 & 5 & 3 \\
\hline 3 & 4 & 2 \\
\hline 4 & 3 & 3 \\
\hline
\end{tabular}

En la encuesta se plantearon dos preguntas abiertas para tratar de localizar, según la opinión de los alumnos, los puntos fuertes y débiles de la experiencia. En cuanto a estos últimos, las aportaciones han sido más bien escasas. El alumno 1 escribió: "resulta desagradable tener que buscar tus errores. Prefiero que lo haga el profesor". El alumno 2 indicó: "en mi caso no he visto que la experiencia me ayudase, aunque puede que funcione en ocasiones posteriores". La alumna 14 manifestó que "sin una ayuda previa, en la que nos expliquen cómo buscar los errores y cómo hacer la propuesta de autorregulación, no es algo útil". En lo que se refiere a las valoraciones de los aspectos positivos de la experiencia, las respuestas de los estudiantes fueron relativamente variadas. Coinciden en destacar la importancia de utilizar los errores como un medio para aprender. Por ejemplo, la alumna 14 indicó: "es bueno apren- 
der de los errores, pues te ayuda a mejorar". En esta misma línea, el alumno 7 comentó que "el sistema de buscar errores te permite aclarar dudas y mejorar en los fallos". La alumna 4 recalcó el hecho de que se trataba de una manera novedosa de trabajar: "nunca había hecho algo así y me ha resultado útil para profundizar en la materia”. Dos alumnos mencionaron que les activó la atención. Por ejemplo, la alumna 5 destacó que "he conseguido mejorar en la atención que pongo al resolver los ejercicios al concentrarme en los errores que encontré previamente". El alumno 10 manifestó una repercusión en su motivación: "la experiencia ha aumentado mi motivación pues me he dado cuenta de que puedo corregir mis errores si me fijo más". La alumna 12 destacó la especificidad del procedimiento: "es interesante el hecho de que personaliza el análisis de errores, lo que permite encontrar soluciones 'a la carta'. Cuando se comentan los errores en general no podemos profundizar tanto en los de cada uno. También es interesante el hecho de que la experiencia nos permitió encontrar una manera individualizada de superar los errores".

\section{SÍNTESIS Y CONCLUSIONES}

Tradicionalmente, la evaluación que realizan los docentes es de carácter sumativo y cuantitativo, es decir, se centra en la valoración del aprendizaje a través de pruebas que son corregidas y facilitadas de vuelta a los alumnos. Sin embargo, este proceder no permite a los estudiantes que extraigan todas las oportunidades de aprendizaje que ofrece la evaluación, puesto que habitualmente su atención se sitúa sobre la nota conseguida. Por otro lado, los errores cometidos suelen tener un carácter sancionador y no se utilizan generalmente como una fuente de aprendizaje.

En este artículo se presentan los resultados de una investigación que se ha llevado a cabo a través de una experiencia de autorregulación en el aula de física de segundo de bachillerato. El objetivo principal de esta investigación ha sido observar y evaluar el proceso de autorregulación de los estudiantes tomando como punto de partida el análisis de la gestión que hacen de la información que aporta una prueba escrita sobre su aprendizaje.

El volumen de trabajo que conlleva la investigación nos ha obligado a restringir el tamaño de la muestra. Aunque este aspecto limita la generalización directa de los resultados, nos ha permitido establecer una primera aproximación al estudio de la competencia con la que los estudiantes utilizan sus errores, lo que constituye un elemento importante en su capacidad de autorregulación.

La experiencia, que ha sido evaluada, en líneas generales, de forma positiva por los estudiantes implicados, nos ha permitido llegar a las siguientes conclusiones:

- La observación de dificultades significativas en la detección y descripción de los errores por parte de los estudiantes nos lleva a pensar que se trata de un aspecto que se debe incorporar a la práctica docente. No podemos dar por sentado que el alumno analiza los errores que comete con la profundidad debida ni que construye de manera espontánea un esquema mental adecuado que le permitirá aprender de ellos.

- Existe una deficiencia patente en las atribuciones causales que hacen los alumnos sobre sus propios errores, lo que repercute no solo en el proceso posterior de autorregulación, sino también en su motivación. Las mayores dificultades se presentan en la descripción de las causas de los errores asociados a las preguntas teóricas.

- La elaboración de una propuesta de autorregulación no resulta una tarea trivial para la mayoría de los estudiantes. En primer lugar, el volumen de trabajo propuesto resulta en numerosas ocasiones muy poco ajustado a la situación del alumno o a los tiempos de los que dispone. Además, en muy pocas ocasiones, el estudiante menciona alguna estrategia específica de aprendizaje que 
se propone utilizar para facilitar la consecución de los objetivos didácticos asimilados deficientemente.

- El proceso de autorregulación se ha demostrado eficaz, tal y como hemos podido observar en los resultados de la segunda prueba de evaluación, especialmente para aquellos alumnos que se han implicado en profundidad. Si bien posiblemente no podamos atribuir esta progresión en su totalidad a la ejecución de la experiencia, el $43 \%$ de los estudiantes ha percibido una relación entre esta y su mejoría. Además, una gran mayoría de los alumnos indica un grado de satisfacción elevado en líneas generales.

- Como era de esperar, tanto en el proceso de detección y descripción de los errores como en la propuesta de autorregulación retroactiva, hemos observado una importante correlación entre el éxito escolar en física y la facilidad con la que los estudiantes llevan a cabo estas tareas.

Tomando estos resultados en consideración, parece evidente la necesidad de que los docentes implementen en sus clases diferentes estrategias para que los estudiantes aprendan a localizar y a analizar sus propios errores y diseñar, a partir de ellos, estrategias adecuadas que les permitan autorregular su aprendizaje. Además, en el transcurso de esta investigación han surgido nuevas preguntas que serán objeto de futuros estudios, como por ejemplo el papel que desempeñan los errores que cometen los estudiantes sobre su proceso de aprendizaje o la relación entre la detección de los errores y el rendimiento académico. Por otro lado, seguiremos profundizando en los factores que influyen en la capacidad de autorregulación de los estudiantes en el contexto de la detección de sus propios errores, buscando su relación con las estrategias cognitivo-motivacionales.

\section{AGRADECIMIENTOS}

A los autores les gustaría agradecer al Dr. José Manuel Suárez Riveiro, de la Universidad Nacional de Educación a Distancia, sus sugerencias sobre la redacción del manuscrito original.

\section{REFERENCIAS}

Allal, L. (1991). Vers une practique de l'evaluation formative. Bruselas: De Boek Univ.

Astolfi, J. P. (1999). El "error", un medio para enseñar. España: Díada Editora.

Buteler, L.; Coleoni, E. y Gangoso, Z. (2008) ¿Qué información útil arrojan los errores de los estudiantes cuando resuelven problemas de física?: Un aporte desde la perspectiva de recursos cognitivos. Revista Electrónica de Enseñanza de las Ciencias, 7(2), pp. 349-365.

ButLER, R. (1987). Task-involving and ego-involving properties of evaluation: Effects of different feedback conditions on motivational perceptions, interest, and performance. Journal of Educational Psychology, 79(4), pp. 474-482.

Cabrerizo, J.; Rubio, M. J. y Castillo, S. (2008). Programación por competencias. Madrid: Pearson Educación.

Escamilla, A. (2008). Las Competencias Básicas. Claves y propuestas para su desarrollo en los centros. Barcelona: Graó.

Hernández-Pina, F.; Sales P.J. y Cuesta, J. D. (2010). Impacto de un programa de autorregulación del aprendizaje en estudiantes de Grado. Revista de Educación, 353 (septiembre-diciembre), pp. 571-588.

Jorbá, J. y SANMARTí, N. (1994). Enseñar, aprender y evaluar: un proceso de regulación continua. Madrid: MEC. 
Latorre, A. (2003). La investigación-Acción. Conocer y cambiar la práctica educativa. Barcelona: Graó. Mammino, L. (2002). Empleo del análisis de errores para aclarar conceptos de química general. Enseñanza de las Ciencias, 20(1), pp. 167-173.

Manson, A. y Singh, C. (2010). Do advanced physics students learn from their mistakes without explicit intervention? American Journal of Physics, 78(7), pp.760-767.

Monereo, C. (2009). Pisa como excusa. Repensar la evaluación para cambiar la enseñanza. Barcelona: Graó.

Nunziati, G. (1990). Pour construire un dispositif d'evaluation formatrice. Cahiers pédagogiques, 280, pp. 47-64.

NúÑez, J. C.; Solano, P.; González-Pienda, J. A. y Rosário, P. (2006). El aprendizaje autorregulado como medio y meta en la educación. Papeles del psicólogo, 27(3), pp. 139-146.

Pintrich, P. R. y Degroot, E. V. (1990). Motivational and self-regulated learning components of classroom academic-performance. Journal of Educational Psychology, 82(1), pp. 33-40.

SuÁrez, J. M. y Fernández, A. P. (2004). Aprendizaje autorregulado: variables estratégicas, motivacionales, evaluación e intervención. Madrid: UNED.

Rosário, P. (2004). Estudar o Estudar: As (Des)venturas do Testas. Oporto: Porto Editora.

Sanmartí, N. (2007). Evaluar para Aprender. Barcelona: Graó.

SCRIVEn (1967). The methodology of evaluation. En R. E. Stake (ed.). Curriculum evaluation. AERA Monograph, vol. 1. Chicago: Rasul McNally.

Shunk, D. (1984). The self-efficacy perspective on achivement behaviour. Educational Psychologist, 1, pp. 199-218.

Yerushalmi, E.; Cohen, E.; Mason, A. y Singh, C. (2012b). What do students do when asked to diagnose their mistakes? Does it help them? I. An atypical quiz context. Physics Education Research, $8(2), 020109$.

Zimmerman, B. J. (2000). Attainment of self-regulation: A social cognitive perspective. En P. R. Pintrich y M. Zeidner (eds.). Handbook of self-regulation. San Diego, CA: Academic Press, pp. 13-39

Zimmerman, B. J. (2002). Becoming a self-regulated learner: An overview. Theory into Practice, 41(2), pp. 64-70.

Zimmerman, B. J. y Paulsen, A. S. (1995). Self-monitoring during collegiatestudying: an invaluable tool for academic self-regulation. En P. R. Pintrich (ed.). New directions in college teaching and learning: Understanding self-regulated learning. San Francisco, CA: Jossey-Bass, pp. 13-27. 


\section{ANEXO \\ Ejercicios y criterios de valoración en la primera prueba propuesta}

Nota: Dejar la pregunta en blanco o no responder correctamente a cada uno de los siguientes aspectos supondrá una calificación de 1 en la pregunta. Se añaden más puntos según los siguientes criterios:

1. Una observación muy importante en la evolución de la astrofísica fue el llamado movimiento retrógrado. Explica en qué consiste y su influencia a lo largo del tiempo

- Describes lo que es el movimiento retrógrado $(+1)$

- Explicas el movimiento retrógrado utilizando el modelo de Ptolomeo, introduciendo los conceptos de epiciclo y deferente $(+1)$

- Presentas el modelo heliocéntrico y señalas que este modelo permite entender el movimiento retrógrado $(+1)$

2. Enuncia y demuestra la segunda ley de Kepler

- Enuncias correctamente la segunda ley de Kepler $(+1)$

- Demuestras matemáticamente la ley de modo riguroso (es decir, con una serie de pasos lógicos que entiendes bien) $(+1)$

- Manejas adecuadamente la notación vectorial $(+1)$

3. La distancia entre el planeta Mercurio y el Sol es de 70,5 millones de kilómetros en su afelio y de 46,5 millones de kilómetros en su perihelio. Sabiendo que su velocidad en el perihelio es de 59,7 $\mathrm{km} / \mathrm{s}$, calcula su velocidad en el afelio.

- Mencionas que la fuerza es central y que eso implica la constancia del momento angular $(+1)$

- Calculas correctamente el momento angular en afelio y el perihelio, respetando la notación vectorial $(+1)$

- Obtienes el resultado numérico correcto: $39,376 \mathrm{~km} / \mathrm{s}$ o $39376 \mathrm{~m} / \mathrm{s}(+1)$

4. Un satélite con una masa de $200 \mathrm{~kg}$ gira alrededor de un pequeño planeta cuya masa es $3,00 \times 10^{24}$ $\mathrm{kg}$ y un radio de $3.000 \mathrm{~km}$, siguiendo una órbita circular. Se pide: a) la velocidad del satélite, b) cuánto tarda en describir una vuelta completa y c) la fuerza gravitatoria que ejerce el planeta sobre el satélite.

- Haces un esquema del problema donde indica las variables que se utilizarán en su resolución

- Aplicas correctamentelasegundaley deNewton para obtener $v=\sqrt{G \frac{M}{R_{P}+b}}$ y $t=\frac{2 \pi}{v}\left(R_{p}+h\right)$ o $T=\sqrt{\frac{4 \pi^{2} R^{3}}{G M}}(+1)$

- Obtienes todos los resultados numéricos correctos con sus unidades $\mathrm{v}=813 \mathrm{~m} / \mathrm{s}, \mathrm{T}=2,34 \mathrm{x} 10^{5}$ s y $\mathrm{F}=0,446 \mathrm{~N}(+1)$

5. Dos masas de 50,0 kg y $200 \mathrm{~kg}$ se encuentran situadas en los puntos A $(-1,0)$ y B $(3,0)$. Encuentra la fuerza que soportaría una tercera masa de $100 \mathrm{~kg}$ situada en el punto $\mathrm{C}(-1,2)$

- Haces un esquema del problema donde representa correctamente las fuerzas $(+1)$

- Conoces el procedimiento de descomposición de fuerzas (llegando o no a los resultados numéricos correctos) $(+1)$

- Aplicas correctamente el principio de superposición a los vectores para llegar al resultado numérico: $\stackrel{1}{F}=5,97 \times 10^{-8} \stackrel{1}{i-1,13 \times 10^{-7} j N(+1)}$ 


\title{
To what extent do high school physics students use their own mistakes to learn? An experience on self-regulation in a secondary school classroom
}

\author{
Ángela Zamora \\ Universidad Nacional de Educación a Distancia \\ angzamora@gijon.uned.es \\ Diego Ardura \\ Colegio Santo Domingo de Guzmán - FESD (Oviedo) \\ d.ardura.ovi@fesd.es
}

Within the competency-based approach for education, the role of the teacher has shifted from acting just as a knowledge transmitter to being a mediator who helps students to be autonomous enough to become the main character of their own learning process. To face this new challenge in education, student's self-regulation of learning seems to be crucial. In this context, how students handle their mistakes is key to prompt the self-reflective phase in the self-regulatory cycle. In turn, this would change the role of mistakes at school so that, instead of avoiding them by all means, students should be able to find and correct their own mistakes to facilitate the rebuilding of knowledge, which is implied in their learning process.

In this paper, we try to get insights on how students manage the information that they extract from a test. The aims of this work are the following: (a) To understand the detection and analysis by students of their own mistakes on a particular test, (b) to analyze the subsequent self-regulatory process through the student's own proposal based on their previous findings, (c) to asses the efficacy of the present learning experience.

The investigation was developed in the context of a high school gravitation lesson plan to 17-year old students in a Spanish high school on their last year within the Physics subject. A total of 14 students participated in the experience. Once the work in the lesson ended, the students took a test to evaluate their knowledge and they were given back their tests with no marking from the teacher. They were asked to find their mistakes, describe them and, with this information in mind, propose a set of activities to overcome their mistakes. The success of the students' performance was evaluated through a second test, which was meant to assess the same topics evaluated in the first one, without the previous knowledge of them. Finally, a questionnaire was used to evaluate the student's degree of satisfaction during the experience.

The high volume of work during the experience made us take a small sample of students. Despite this, the present investigation led us to establish a first approach on the study of how students deal with their mistakes, which constitutes a basic aspect in their self-regulation behavior. This investigation reached several conclusions: First, we have found serious deficiencies in the way the students find and explain their mistakes. Second, the student's attributions to the mistakes are often poorly described, which makes the proposal of self-assignment not a straightforward task. The students rarely propose any specific learning strategies that help them understand the materials. Third, the self-regulation process seems to be effective for students who showed a high level of compromise during the different sessions. Even though we cannot attribute all the progression detected in the second test to the experience, $43 \%$ of students perceived a relationship between their performance and the work with their mistakes. Finally, as expected, we have found a relationship between the school performance of students in Physics and both the process of finding and describing the mistakes and the self-regulation proposal.

Taking our results into account, we point out the need of implementing strategies in the classroom not only to help students analyze their own mistakes, but also to use them as a means to learn in the subsequent decisionmaking process. Finally, in the course of our investigation several questions have emerged which will be the object of future research. 